\title{
The Influence of Longitudinal Stiffeners on Weld Toe Stress Concentrations in Multi Planar Tubular KK joints
}

\author{
C. O. Woghiren ${ }^{1, a}$ and F. P. Brennan ${ }^{2, b}$ \\ ${ }^{1}$ Mobil Producing Nigeria Unlimited, Room 6B 28A, Mobil House, Lagos, Nigeria. \\ ${ }^{2}$ School of Engineering, Whittle Building, Cranfield University, Cranfield, Beds, MK43 OAL, UK. \\ ${ }^{a}$ charles.o.woghiren@exxonmobil.com, ${ }^{b}$ f.brennan@cranfield.ac.uk
}

Keywords: Tubular Joints, Stiffened Tubular Joints, Multi Planar Tubular Joints, Welded Tubular Joints, Stress Concentration, Parametric Equations.

\begin{abstract}
This paper reports a parametric stress analysis of various configurations of rack plate stiffened multi-planar welded KK joints using the finite element method. The KK joint finds application in the leg structure of offshore Oil \& Gas jack-up platforms. The rack plate is a dual purpose element of the joint because it firstly functions as a stiffener which reduces the stress concentration at the brace/chord intersection. This could be an immense contribution to the increase in fatigue life of the joint but other hot spot sites are introduced to the joint. The rack is also used for raising and lowering of the jack-up hull which gives the jack-up platform its jacking capability. Over 120 models using a combination of shell and solid elements were built and analysed within ABAQUS. Non-dimensional joint geometric parameters; $\beta, \gamma$ and $\Omega$ were employed in the study with $\Omega$ being defined as the ratio of rack thickness to chord diameter. Stress Concentration Factors (SCFs) were calculated under applied axial and OPB (out-of-plane-bending) loading. Three critical SCF locations were identified for each load case, with each location becoming the most critical based on the combination of the non-dimensional parameters selected for the joint. This is important as careful design can shift the critical SCF from an area inaccessible to NDT to one that can be easily inspected. The SCF values extracted from the models were used to derive six parametric equations through multiple regression analysis performed using MINITAB. The equations describe the SCF at the different locations as a function of the non-dimensional ratios. The equations not only allow the rapid optimisation of multi-planar joints but also can be used to quickly identify the location of maximum stress concentration and hence the likely position of fatigue cracks. This in itself is an invaluable tool for planning NDT procedures and schedules.
\end{abstract}

\section{Introduction}

Longitudinally stiffened tubulars find application in the legs of jack-up platforms. The stiffeners are usually found as rack plates which aid in the raising and lowering of the jack-up via a rack and pinion mechanism. A survey of available literature reveals that considerable research effort has been directed towards an understanding of the effect of internal ring stiffeners in tubular joints as opposed to the effect of longitudinal stiffeners, nevertheless the rack plate has been observed to reduce the stress concentration at the brace/chord intersection of stiffened joints. Stiffeners which reduce the stress concentration in certain locations are suspected of introducing hot spots in other locations and this indeed justifies the need for research into the effect of rack plates in jack-up chords.

Extensive stress analyses of uni-planar tubular joints have been carried out in the past while most studies of multi-planar joints have focused on an ultimate strength analysis. Furthermore, the study of the effect of stiffeners has mainly focused on their application to uni-planar joints. This parametric study involves a stress analysis of a rack plate stiffened multi-planar KK joint as shown in Fig. 1 below. 


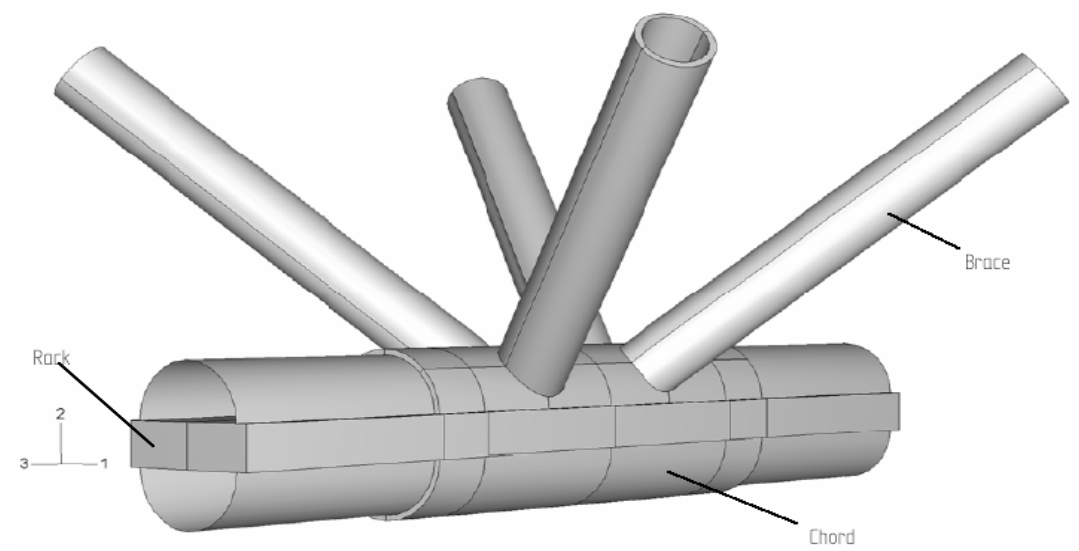

$$
\begin{aligned}
& D=\text { chord diameter } \\
& \mathrm{z}=\text { rack thickness } \\
& \mathrm{d}=\text { brace diameter } \\
& \mathrm{T}=\text { chord wall thickness } \\
& \Omega=\mathrm{z} / \mathrm{D} \\
& \beta=\mathrm{d} / \mathrm{D} \\
& \gamma=\mathrm{D} / 2 \mathrm{~T}
\end{aligned}
$$

Fig. 1: 3D view of a stiffened KK tubular joint.

The stiffened multi-planar KK joint under investigation is an Integral part of the lattice legs of a jack-up platform. Each leg is a critical component of the jack-up as failure of one leg will lead to the collapse of the whole structure. An extensive understanding of possible crack sites in the KK joint, is achieved by this study and is a first step to prevent an eventual collapse of the platform due to fatigue cracking.

A finite element stress analysis of over 120 models utilising a combination of 3D solid elements and 2D shell elements was employed for the purpose of this work with the ultimate goal of developing parametric Stress Concentration Factor (SCF) equations to represent the hot spot stress at the brace/chord intersection and any other location identified as a potential hot spot. The study has been restricted to axial and out-of-plane bending (OPB) loading modes while only 3 nondimensional joint geometric parameters; $\beta, \gamma$ and $\Omega$ were varied.

The circular chord in Fig. 1 above was split to accommodate the continuous rack plate and is described as the opposed pinion leg configuration of a jack-up wherein the KK joint is repeated through out the legs. Other leg configurations exist, but this study focuses on the split tubular with continuous central rack. In-plane bending (IPB) loads were not considered in this study because of the negligible effect of the stiffener on the SCFs under IPB as identified by Myers et al [1]. Other non-dimensional parameters by which a tubular joint can be described exist but the nondimensional parameters selected for this study are believed to have the greatest effect on the SCFs in the joint under investigation.

\section{FEA and Parametric Study}

The details of the FE analysis including mesh optimisation, refinement and convergence analyses can be found in reference [2]. Several studies were conducted to verify the approach used against published solutions for uni-planar tubular joints [3, 4]. Fig. 2 shows an example of a stress contour plot of a stiffened KK joint under OPB. Fig. 3 illustrates the parametric matrix of FE models analysed and the basis for the resulting parametric equations. 


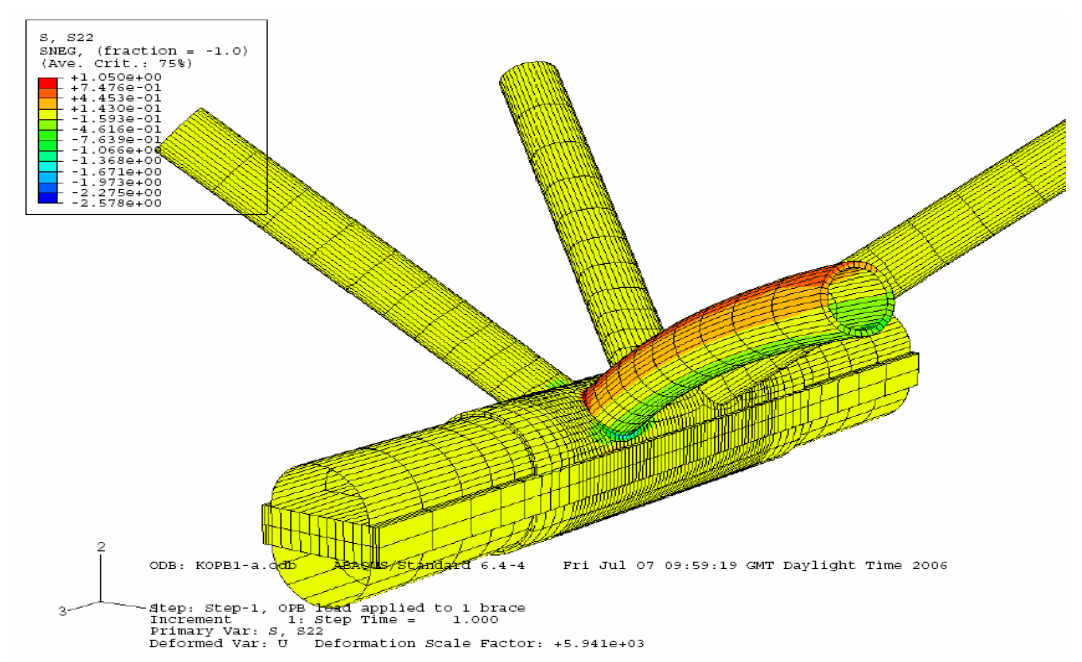

Fig. 2: Contour plot of a stiffened KK joint under OPB.
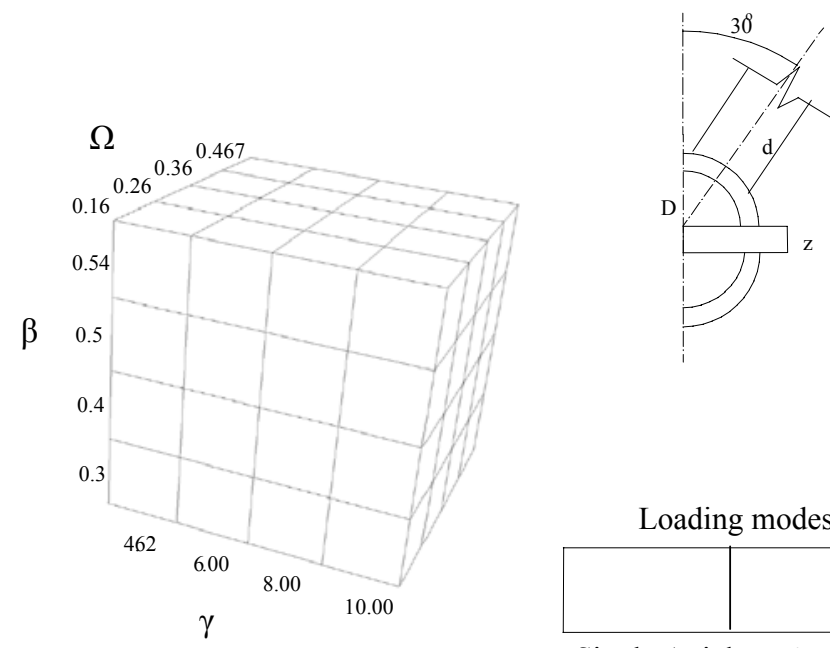

Fig. 3: Parametric matrix of FE models.

\section{Parametric Equations}

The SCFs extracted from each model were input into MINITAB for regression analysis while the non-dimensional parameters were supplied as predictor variables. The equations produced were arrived at by trial and error. The first sets of trials were aimed at achieving model equations with good fit and minimal deviation from the data points (SCFs). However, the relevance of this study to design necessitated that the equations should be conservative rather than to simply provide a best fit. This was achieved by assessing the performance of the initial equations and then marking up the FEA SCFs at all non-conservative points and marking down if required at conservative points. The regression analysis was then performed with the refined data. The final equations are given below.

$$
\begin{aligned}
\text { SCFA-CRi }= & -9.518-3.273 \beta-19.619 \Omega+12.240 \exp (\Omega)-0.0321 \gamma 2+1.86 \beta \gamma+14.892 \beta \Omega+ \\
& 0.368 \gamma \Omega-2.879 \beta \gamma \Omega \\
\text { SCFA-BCc }= & 72.9-62 \beta+19.5 \gamma-2.60 \Omega+34 \beta 2.4-35.9 \gamma 0.85+3.37 \Omega 2-49.6 \gamma-1 \beta-1+6.90 \gamma-1 \beta-2 \\
& +8.8 \beta \gamma 0.85-7.0 \beta 2.4 \gamma 0.85+5.35 \gamma 0.65 \beta 12.5 \exp -(\gamma-10)
\end{aligned}
$$


SCFA-CBs $=3.50-2.11 \Omega+1.88 \Omega 2-12.6 \beta 1.2 \gamma-1+0.0262(\beta-1.2 \gamma) \exp (\beta 1.2 \gamma-1)$

$\mathrm{SCFB}-\mathrm{CRe}=0.798-5.056 \beta-0.207 \gamma+0.185 \Omega+1.405 \beta \gamma$

$\mathrm{SCFB}-\mathrm{CBi}=-0.972+2.81 \beta+0.443 \gamma-0.213 \Omega-0.811 \gamma \beta 2-0.0168 \gamma \beta-2$

The equations above are valid for the entire range of non-dimensional parameters investigated i.e. $0.3 \leq \beta \leq 0.54,4.62 \leq \gamma \leq 10,0.167 \leq \Omega \leq 0.467$

It was impossible to fit a good quality equation to the entire database for SCFB-BCc hence a reduction in the validity range was imposed for this location.

$\mathrm{SCFB}-\mathrm{BCc}=3.2+4.9 \beta+0.441 \gamma-3.4 \exp (\beta)-0.0714 \beta 0.6 \gamma 2+0.0327 \gamma 1.65 \beta 24.5 \exp -(\gamma-20)+$ $0.20 \beta 2 \Omega 2$

$0.3 \leq \beta \leq 0.54,4.62 \leq \gamma \leq 6,0.267 \leq \Omega \leq 0.467$

' $A$ ' refers to Axial load, ' $\mathrm{B}$ ' is $\mathrm{OPB}$, ' $\mathrm{CR}$ ' is the chord side of the chord/rack intersection, ' $\mathrm{CB}$ ' is the chord side of the chord/brace intersection, ' $\mathrm{BC}$ ' is the brace side of the brace/chord intersection, ' $\mathrm{i}$ ' is the internal location, ' $\mathrm{c}$ ' is the crown location and ' $\mathrm{s}$ ' is the saddle location.

\section{Discussion, Summary and Conclusions}

A systematic parametric study covering various configurations of Jack-up Platform rack plate stiffened multi-planar KK joints was conducted using finite element analysis. 120 models defined by non-dimensional joint geometric parameters; $\beta, \gamma$ and $\Omega$ were employed in the study with $\Omega$ described as the ratio of rack thickness to chord diameter. The finite element procedure was verified against results for unstiffened tubular joints published in the available literature.

Three critical SCF locations were identified for each load case, with each location becoming the most critical based on the combination of the non-dimensional parameters selected for the joint. The six equations describe the SCF at different critical locations as a function of the nondimensional ratios. In all cases, the final equations are conservative for more than $95 \%$ of the models analysed. These new design equations not only allow the rapid optimisation of multi-planar joints but also can be used to quickly identify the location of maximum stress concentration and hence the likely position of any fatigue cracks. This in itself is an invaluable tool for planning NDT procedures and schedules. It can be appreciated that where the maximum SCF is situated internally, inspection becomes impossible and design penalties by certification authorities are likely. The equations developed here allow subtle changes in stiffener geometry to shift the critical SCF to external locations so that inspection becomes possible.

Future work will involve experimental stress analysis of tubular joint specimens in order to verify the FEA based observations. The validity range could be extended for design purposes and the influence of other non-dimensional parameters investigated. There is immense scope to develop the idea of designing the location of maximum SCF for ease of inspection.

\section{References}

[1] Myers P. T., Brennan F. P. and Dover W. D: Marine Structures 14, 485-505, 2001.

[2] Woghiren C.: MSc Thesis, University College London, 2006.

[3] Chang E. and Dover W. D.: Int. J. of Fatigue Vol. 18, No. 6, pp. 363-387, 1996.

[4] Hellier A. K., Connolly M. P. and Dover W. D.: Int J of Fatigue 12, No 1, pp 13 - 23, 1990. 